\title{
Dante e la poesia italiana del Novecento: il caso Quasimodo
}

\section{Andrea Ciccarelli}

In un saggio dedicato alla presenza di Dante nella letteratura del nostro Novecento, uno dei critici più sensibili al problema, riepilogando gli ostacoli che solitamente s'incontrano nel corso di una tale analisi, proponeva all'attenzione del lettore una riflessione: "Difficoltà del dantismo dei poeti contemporanei: potrebbe essere il titolo di una ricerca" (Petrucciani 164). Che la poesia italiana proceda piuttosto da Petrarca che da Dante e cosa tutto ciò abbia significato per la nostra tradizione letteraria, è stato ampiamente dibattuto e, non a caso, con maggior lucidità proprio da quei poeti che in qualche modo si sono misurati con l'esperienza dantesca. ${ }^{1}$

Non sarà certo superfluo ricordare, comunque, qualcuna delle conseguenze derivate da questa scelta, attraverso la voce di uno di questi poeti:

Accettando Petrarca la nostra letteratura si trovò, fin dai suoi inizi, a compiere delle capitali definitive rinunzie ... la poesia italiana è stata dopo Petrarca privata dell'orgoglio della scoperta, dei contatti più freschi e magari più bruschi dell'anima con le circostanze episodiche della vita ... dopo la Commedia non vi fu in Italia se non eccezionalmente il gusto della narrazione, questa presa di possesso della realtà . . . quell'attenzione e quella fiducia nei particolari e nei frammenti del mondo . . . escluse per sempre dall'inquietudine interiore del Petrarca . . . . Il Petrarca dominò, vinse fino all'annientamento completo una materia che poi fu quasi naturale evitare, senza più combatterla e spesso senza più considerarla .... Si pensi quindi quanta parte dell'esistenza è rimasta esclusa, dopo di lui, dal dominio della letteratura ... e si pensi quanto è costato Petrarca a chi doveva in ogni modo averlo fra i propri numi tutelari. (Luzi 22-5)

I motivi che hanno sollecitato, nel Novecento, la prosecuzione di una tale situazione culturale, si possono schematicamente riassumere nell'impossibilità di riacquistare la sicurezza ideologica che ha consentito al Dante "poeta, filosofo, teologo, scienziato" (Bo, "Dante" 193) di costruire una struttura poetica unitaria e compatta, basata su un razionalismo che "resta estraneo ai nostri tempi . . perché pone i suoi significati nei fatti e non nelle idee. Ed è proprio la ragione dei fatti che oggi ci sfugge" (Montale 33). Una ragione difficile da svelare perché, forse, come ha suggerito Ungaretti, siamo bloccati di fronte al fatto che in Dante la poesia scaturisca dal "sacro, purtroppo quasi interamente inibito alle nostre monche esperienze" (941). In altre parole, la condanna ad accostarsi a Dante con la coscienza di non poterne ripercorrere l'esperienza, a meno di inopportune forzature ideologiche: 
Dante non può essere ripetuto .... . Poeta concentrico, Dante non può fornire modelli ad un mondo che si allontana progressivamente dal centro e si dichiara in perenne espansione. (Montale 33)

"Difficoltà" riconosciute dagli stessi poeti-e non minori-del nostro Novecento quindi, ma che lasciano pur sempre spazio alle eccezioni (ed è stato giustamente ricordato che fra queste, proprio chi ha asserito la non ripetibilità dell'esperienza dantesca, Montale, è forse quello più volte accostato dalla critica al nome di Dante: cfr. Petrucciani 164). Ma è stato altrettanto giustamente detto che una difficoltà di fondo, una "resistenza non superficiale" (Jacomuzzi 217) ad avvicinare il nome di Dante ad un poeta del Novecento, esiste anche per la critica. "Al di là del valore esemplare e della suggestione dei temi e della scrittura" che l'opera dantesca suscita in molti autori contemporanei, possiamo parlare di "un uso allegorico del linguaggio letterario, cioè l'idea e la prassi che stanno a fondamento della poesia dantesca"? (Jacomuzzi 217). Insomma, l" "eccezione" novecentesca, pur non potendo attuarla, può almeno intuire e utilizzare l'idea di poesia così come la concepiva Dante, oppure si assiste ad una semplice ripresa di modi e temi? Nell'impossibilità di enumerare un cospicuo gruppo di nomi (Montale, ancora, è stato suggerito da Jacomuzzi; ma mi sembra che per altri, per quanto profondo e basilare sia il loro rapporto con Dante, non si possa applicare la controprova del criterio allegorico di cui sopra) credo non rimanga che rassegnarsi ad una lettura che, una volta messa a nudo l'entità dei risultati, proceda all'analisi delle cause che hanno originato tali riprese dantesche.

Il caso (l'eccezione) che qui si propone, Quasimodo, può forse sorprendere perché per le sue origini ermetiche indurrebbe a pensare più alla linea $\mathrm{Pe}$ trarca-Leopardi che non a Dante, benchè lo stesso autore, nelle sue opere saggistiche, si sia richiamato apertamente al magistero stilistico e morale dell'Alighieri. ${ }^{2}$ In realtà, che Quasimodo abbia attivamente messo a profitto la lezione stilistica di Dante, soprattutto quella infernal-petrosa, è stato recentemente dimostrato (cfr. Scorrano e Ciccarelli). Qui, forti anche di queste verifiche filogiche, si vuole piuttosto ricostruire l'itinerario ideologico che conduce il poeta siciliano a quella che, almeno sotto l'aspetto teorico, appare una tarda riscoperta di Dante. ${ }^{3}$

Questo riavvicinamento a Dante assume un rilievo particolare perché viene a coincidere con un periodo culturale (1945) in cui il poeta siciliano - già indicato da molti come un indiscusso protagonista dell'ermetismo-si propone all'attenzione critica come uno dei più accesi sostenitori della corrente neorealista. Non c'è bisogno di ricordare, credo, come negli anni trenta, già con Acque e Terre (1930) e poi soprattutto in Oboe sommerso (1932) e Erato e Apollion (1936), Quasimodo avesse privilegiato una poetica (la cosiddetta "poetica della parola") ${ }^{4}$ che nei suoi aspetti esteriori favoriva il ripiegamento su se stessi, la tendenza a risolvere il discorso lirico su un piano soggettivo. Le poesie maturate durante e dopo il secondo conflitto mondiale mostrano 
invece il tentativo di farsi voce corale, di tramutare le proprie riflessioni liriche in dialogo. ${ }^{5}$ I motivi di quest'apertura verso le tematiche civili sono stati riassunti da Quasimodo come una necessaria riconsiderazione del ruolo del poeta e della poesia in una società traumatizzata dalle ferite lasciate aperte dalla guerra: ${ }^{6}$

Oggi, poi, dopo due guerre nelle quali l'“eroe" è diventato un numero sterminato di morti, l'impegno del poeta è ancora più grave, perché deve "rifare" l'uomo. (23-4)

La ricerca lirica di Quasimodo, venuta in contatto con l'esperienza bellica, avrebbe quindi subito un naturale ampliamento delle proprie tematiche esistenziali, facendosi portavoce di esigenze collettive, perché le "domande che il poeta pone a se stesso", le pone "a tutti" (13). Tutto ciò non può non riflettersi sul suo rapporto con Dante, visto che i primi segnali di esso, come s'è detto, coincidono proprio con questa nuova impostazione ideologica. Quasimodo si batte per la "fioritura di una poesia sociale", una poesia che si rivolga "ai vari aggregati della società umana", (40) ed è chiaro, perciò, che il confronto con la propria tradizione letteraria non verterà su questioni di "contenuti". È la guerra che mutando "la vita morale d'un popolo . . . richiama con violenza un ordine inedito nel pensiero dell'uomo, un possesso maggiore della verità: le occasioni del reale incidono nella sua storia" (34-5). Verità e reale, quindi, saranno i necessari punti di riferimento delle nuove poesie. Si tratterà allora di ricercare un linguaggio poetico che aiuti a realizzare quel "desiderio di discorso", quei "movimenti larghi di ritmo e di forme", quella volontà poetica che "aspira al dialogo e non al monologo" (40). E questo linguaggio (non potendo più essere quello che racchiudeva le proprie verità "nei simboli e nei barocchi petrarcheschi" 38), è individuato da Quasimodo nella "durevole forza del semplice stile" di Dante (44). È questo il "linguaggio visivo e concreto" (138) di cui la poesia contemporanea ha bisogno per spezzare le catene del "decoro ritmico" petrarchista che "ha dettato ancora solitudine e rassegnazione e indifferenza" nell'ultima poesia europea (124). Ripetiamo, Quasimodo si muove all'interno di una ricerca stilistica. Uno stile che si avvicini il più possibile alle esperienze di chi, dopo l'inferno bellico, si ripropone di "rifare l'uomo". Ecco che il contatto con la concretezza del Dante infernale diventa quasi naturale visto che Quasimodo, dopo aver affermato che "l'Inferno è il luogo dell'uomo nella sua contrastante natura" (134), termina il saggio sull'Alighieri del ' 52 asserendo che "la nuova generazione sa che per ritrovare l'uomo non deve incunearsi ancora nell'inferno: l'inferno è qui" (139). Consonanza di temi e di stile, dunque. Ma è una ricerca che non sembra proseguire oltre i confini infernali. Al Quasimodo che intitola le sue liriche Colore di pioggia e di ferro, Auschwitz, Ancora dell'inferno, non interessa il Dante "cifra oscura di sottofondi e allusioni" (138), quello che nel Paradiso ritorna a Beatrice e alla Vita nuova, che affonda le sue "radici in un tempo inventato" e non nel reale (126). 
Che Quasimodo cadesse in pieno nel diffuso pregiudizio che la "parola realistica" della Commedia non risuoni nelle ultime due cantiche, non dovrebbe sorprendere più di tanto, visto che il recupero di Dante è giocato all'interno di questo parallelo inferno=guerra. ${ }^{7}$ Seguendo il filo logico della sua riduttiva interpretazione del poema dantesco, si può anzi dire che se Quasimodo accettasse la poesia elegiaca del Paradiso incorrerebbe in una contraddizione stilistica, richiudendosi nuovamente in quella sfera di simbolismi che allontanano dalla verità. D'altra parte, il poeta, non nasconde che per lui "la decadenza della cultura cristiana è in atto" (122), e quindi difficilmente si può accostare al Paradiso senza valutarlo una "fuga" dalla realtà, un tentativo ormai impraticabile per l'uomo moderno che "ha imparato a sottrarsi alle metafisiche" (123). E Quasimodo non sembra lasciare spazio ad alternative, quando, non a caso prendendo ad esempio Eliot, afferma che il poeta di lingua inglese ha finito per impigliarsi in "richiami culturali di civiltà lontane", proprio per non essersi attenuto all'unico insegnamento che Dante poteva offrirgli: "La lezione di Dante per Eliot poteva essere una sola; ed era lezione di linguaggio, di poesia" (138).

Il poeta siciliano non pare preoccuparsi né ammettere l'eventualità che l'autore della Commedia possa penetrare nelle poetiche novecentesche autonomamente, al di fuori di questi schemi restrittivi. Un'impressione confermata dall'asserzione che gli uomini come Dante sono eletti "di volta in volta cittadini contemporanei del mondo" non per "memoria o amore", ma per "l'agonia d'una cultura, la sinuosa corruzione delle forme poetiche e di un linguaggio" (122). La ripresa dell'esempio dantesco, perciò, prescinde da ogni tentativo di capire a fondo i meccanismi della sua poetica e del suo realismo fondato non soltanto sulla realtà terrena, ma che si nutre anche "dell'invenzione, del sogno, della visione, della religione" (Bárberi Squarotti 171). Un Dante dimidiato, che per ragioni non meno storiche che estetiche (la "corruzione delle forme poetiche" contemporanee) finisce per rientrare in un piano di recupero funzionale ad una determinata situazione culturale. (E questa funzionalità, tutto sommato, è il segno di parte di quelle difficoltà ideologiche - anticipate in apertura-che la poesia contemporanea incontra nell'avvicinarsi alla lezione dantesca).

Date queste premesse e considerato che Quasimodo ambiva ad "essere salutato come profeta e capofila di quella poesia nuova che . . aspira al dialogo" (Petrucciani 176) non c'è da stupirsi che abbia intrecciato con frequenza i suoi giudizi su Dante con gli sviluppi delle polemiche suscitate dalle sue liriche "civili". ${ }^{8}$ Anzi, il richiamo al linguaggio concreto di Dante finisce per scadere, alle volte, in uno scoperto parallelismo:

Il dramma c l'epica sono le aspirazioni che la nuova generazione piega verso il suo dolore morale ... O Oggi, nel silenzio della poesia italiana . . . un ritorno alla parola realistica di Dante allontancrà lo sfocato, barocco petrarchismo. (139)

Inutile dire che affermazioni come queste, in una lectura Dantis tenuta a 
Firenze nel '52, non possono che essere rivolte e nemmeno troppo velatamente a quella "scuola ermetica" che l'anno successivo, nel Discorso sulla poesia, verrà definita "estremo antro pastorale fiorentino di stilemi metrici" (35). Dante poeta-simbolo per chi affronta il reale ed esce dal suo "antro" per parlare al mondo, dunque, in opposizione agli epigoni del poeta che si chiude in se stesso, il lirico da cui dipende gran parte della nostra poesia e a cui però è negata la "possibilità d'una proiezione oggettiva del mondo" (115). Dante entra nella poetica di Quasimodo come l'anti-Petrarca, insomma, nei limiti in cui Petrarca finisce incolpevolmente per rappresentare, nei secoli, l'inesauribile fonte di una poesia che interpreta il mondo in funzione del proprio io e non viceversa. ${ }^{9}$

Altrove l'accenno a Dante è ancor più scopertamente apologetico, soprattutto se si considera che proviene dal discorso tenuto nel '59 a Stoccolma in occasione del ritiro del premio Nobel:

Dante ... accanto alle dolcissime poesie ermetiche della scuola del dolce stil novo più tardi aggiungerà, senza tradire la sua integrità morale, la violenza delle invettive umane e politiche, non dettate dall'odio ma dalla giustizia interna e religiosa in senso universale. $(68)^{10}$

Il fine di questo brano è talmente evidente che diventa superfluo ogni commento. Che senso avrebbe definire "ermetiche" le poesie giovanili di Dante, nonché l'affermare che l'Alighieri nel passare al realismo della Commedia non ha tradito la sua integrità morale, se non per gettar luce sulla vicenda Quasimodo? Eppure, se non ci si lascia influenzare dal facile strumentalismo, non è impossibile identificare alcuni dei luoghi in cui quelle invettive dettate da giustizia e non da odio risuonano nelle liriche di Quasimodo. Si pensi, ad esempio, alla poesia de Il falso e vero verde intitolata jacoponicamente Laude, in cui un Figlio (assassinato) e una Madre dialogano sulla violenza prodotta dalla recente storia italiana e sul naturale nonchè brutale atto di contrappasso collettivo in cui è culminata (la folla inferocita che infierisce sul cadavere di Mussolini a piazzale Loreto). Si rifletta su quanto affermato dal Figlio: "l'ira e il sangue / colano giustamente" e si vedrà che il collegamento con quelle invettive "umane e politiche", per quanto strumentale, non è giocato soltanto ad un livello di esemplarità apologetica.

In alcuni casi, poi, certe asserzioni sulla vita di Dante lasciano trasparire la volontà di illuminare tratti della biografia dantesca sottolineandone quegli aspetti in qualche modo condivisi anche dallo stesso Quasimodo: "Nel rumore delle lotte di parte, [Dante] riprende le antiche letture di Stazio, di Ovidio, di Virgilio, di Orazio" (129). Come non pensare al Quasimodo che, per sua ammissione, sotto i bombardamenti, "nei giorni della furia tedesca e latina", traduceva Virgilio, Catullo, Omero? (109) E ancora: "Con lo studio degli antichi poeti Dante supererà la corrente lirica del volgare apologetico e continuatore d'un sentimento uniforme" (129). 
In quel "sentimento uniforme" da superare, non è difficile leggere la poetica dell'ermetismo che Quasimodo asserisce di trasformare in canto, anche lui grazie alla lezione di quegli antichi poeti:

Virgilio, Omero, Catullo, Eschilo, Ovidio . . . . Anni di lente letture per giungere ... dalla prima approssimazione ... della parola al suo intenso valore poetico. Non nel corpo di una "poetica della parola", ma in quello della sua concretezza, della rappresentazione visiva, in funzione del suo linguaggio diretto e concreto. (32)

Linguaggio "diretto", "concreto", "visivo". Sono gli attributi del linguaggio del reale di Dante cui le nuove generazioni poetiche devono guardare per "aspirare al dialogo", come s'è visto. Ma proprio quando questo rapporto con Dante pare esplodere in dichiarazioni che suonano più programmatiche che spontanee, mostra invece di poggiare le sue radici su movenze sincere, non importa quanto ingenuamente ritenute davvero fondate su una comune matrice culturale. Mi riferisco al ruolo di Virgilio, il più amato degli antichi se Quasimodo firmava per anni le sue lettere d'amore con il nome del "silenzioso e casto" poeta delle Georgiche."

Virgilio è visto come il padre spirituale e poetico di entrambi: del siculo-greco Quasimodo che, grazie a Virgilio, arriva "al discorso, ad una misura d'oggettivazione" (109); e del pellegrino medioevale che sull'autore dell'Eneide "impara il dialogo", apprende come spostarsi "dalla poetica della memoria a quella del reale, degli oggetti" (133). Ancora un riferimento laterale alle polemiche contemporanee al poeta, quindi (memoria=intimismo=ermetismo, vs la triade dialogo-reale-oggettività). Ma, come è stato già suggerito (Ciccarelli 420-2), il sentimento provocato da questa comune discepolanza ha recato frutti concreti. Basti ricordare la poesia Dialogo de La vita non è sogno, in cui Quasimodo intreccia il tema dell'ade virgiliano (il mito di Orfeo ed Euridice), con quello "reale" dell'inferno dantesco. Così, all'incipit tratto dalle Georgiche ("At cantu commotae Erebi de sedibus imis / umbrae ibant tenues simulacraque luce carentum”), fa subito coro un al di là infernal-petroso, che trasporta le "umbrae ... tenues" dell'antico mito in un contesto concretizzato da un lessico inequivocabilmente dantesco:

Siamo sporchi di guerra e Orfeo brulica d'insetti, è bucato dai pidocchi, e tu sei morta. L'inverno, quel peso di ghiaccio, l'acqua, l'aria di tempesta furono con te...

ma il nostro tempo è stato furia e sangue:

altri già affondavano nel fango, avevano le mani, gli occhi disfatti, urlavano miscricordia e amore.

Abbiamo evidenziato nel testo i termini di sicura provenienza infernale (mi riferisco, è ovvio, alla "piova / etterna, maladetta, fredda e greve" che sotto 
forma di "grandine grossa, acqua tinta e neve / per l'aere tenebroso si riversa" e fa "urlare" come cani i golosi all'inizio del sesto canto, nonché al fango bollente dello Stige dove sono puniti gli iracondi). Ma è chiaro che l'intero brano riecheggia il mondo senza speranza dell'Inferno dantesco. Un mondo in cui il messaggio poetico si esplica in modo "diretto" e "visivo", senza essere attenuato dalla grazia del "mansuetus" Virgilio: "Vidi genti fangose in quel pantano, / ignude tutte, con sembiante offeso (Inf. 7.110-1). Virgilio come comune maestro di poesia, quindi, ma Dante come un compagno di viaggio ideale, come un alunno più anziano che avendo già fatti propri gli insegnamenti dell'antico, ci mostra la strada per renderli attuali.

Il rapporto con Dante, perciò, filtrato o meno attraverso la lezione dei classici, apologetico o strumentale che lo si voglia dire (e abbiamo anticipato appositamente dei passi che non lasciano ombra di dubbio su una tale utilizzazione), svolge un suo preciso ruolo nella poetica post-bellica di Quasimodo. Ed è proprio su questo ruolo che, forse, vale la pena di soffermarci ancora.

Ł̀ fin troppo chiaro che l'interesse per Dante non nasce all'insegna del fascino esercitato dal plurilinguismo dantesco, se per plurilinguismo s'intende la dichiarata curiosità per una vasta sperimentazione stilistica. Allo stesso tempo, però, se Quasimodo insiste per il "linguaggio del reale", per una poesia sociale, non è per sostenere una prevalenza di "contenuti"; al contrario, sono "proprio le ragioni formali . . . a comprendere la nostra poesia nella partecipazione umana del mondo" (40). È proprio lo stile (il "semplice stile" di Dante) a caratterizzare i contenuti, non l'opposto. Il poeta affida il proprio messaggio sociale ad un determinato modo di far poesia, una poesia che per la sua apertura al dialogo manifesta immediatamente le sue intenzioni estetiche. Il formarsi di questa poetica - che è poi il movente che conduce Quasimodo a Dante - s'intuisce già in un saggio su Petrarca del ' 45 (e quindi prima che il poeta, nel biennio '52-3, s'impegni pubblicamente ad indicare alla poesia italiana la via realistica di Dante), là dove un Quasimodo che è nel pieno della sua riforma poetica asserisce che il reale e l'oggetto sono assenti dalla lirica petrarchesca, perché Petrarca "parla con la voce del sentimento della solitudine" (118). Siamo in quello stesso 1945 in cui il poeta, in un breve scritto dal titolo Cultura e politica, nell'ammonire i giovani a non cadere nelle trappole di un'arte anonima e falsamente collettiva, afferma che questi stessi giovani che si affacciano alla poesia, dopo aver "conosciuto la morte e il lutto . . ora vogliono conoscere la 'vita' della propria terra" (270). Si comincia a profilare quella necessità di apertura formale che poi si materializzerà nell'opposizione al simbolismo ermetico (o a ciò che Quasimodo vede come tale). È chiaro, infatti, che per evitare che la poesia si risolva in un esercizio teso solo a lenire il dolore di chi scrive, è necessario uscire da una poetica che si affida a simboli più o meno oscuri per dispiegarsi invece nel canto $\mathrm{e}$ gettare luce su quella "vita" che si desidera conoscere. Petrarca o, meglio, ciò che ha rappresentato per la tradizione italiana, non pare quindi poter rien- 
trare nell'ottica di chi assegna un tale compito alla poesia. Quasimodo non fa mistero che la ricerca di una nuova via implica la convinzione del fallimento delle poetiche novecentesche: "il surrealismo è morto, forse, nella stessa ora in cui da noi tramontava l'ermetismo" (27). Con la guerra e la susseguente distruzione di ogni sicurezza ideologica e formale, la "nuova generazione ... s'è trovata improvvisamente senza maestri apparenti per poter continuare a scrivere poesia" (39). Questa mancanza di modelli, ovviamente, va sempre considerata dal punto di vista di Quasimodo, il quale non si stanca di ripetere il proprio credo realistico: "l'uomo vuole la verità dalla poesia" (28). È il momento storico a spingere il poeta ad interrogarsi, a guardarsi dentro, non il proprio innato "sentimento della solitudine". Ma guardarsi dentro significa anche cominciare a valutare il peso della tradizione letteraria che ci ha preceduto e che ha prodotto quelle poetiche ormai inattuali. E quando nel ' 46 , nel primo dei suoi "Discorsi sulla poesia contemporanea," Quasimodo comincia a porsi il problema, Dante è escluso da questa ricerca, non sembra poter colmare quel vuoto di "maestri" causato dal fallimento del lirismo di marca petrarchesca: "Per la poesia, la filosofia di Dante è crollata . . . Dante non è un poeta amato dai contemporanei" (22). È la prima volta che il nome di Dante compare in un saggio dedicato da Quasimodo alla poesia del '900 ed è significativo che il poeta, il quale pochi anni dopo inviterà a leggere la $\mathrm{Com}$ media per cancellare lo "sfocato barocco petrarchismo", sottolinei lo scarso amore dei contemporanei per Dante. Quasi ad anticipare la necessità di quello che, una volta scoperta l'attualità della lezione dantesca, diverrà un incessante ritornello: leggere Dante per dimenticare Petrarca. Nel '46 Quasimodo ha già implicitamente espresso i suoi dubbi sulla validità dell'insegnamento petrarchesco per la poesia italiana, assetata di quel "reale" assente dall'opera del grande lirico trecentesco. Ma di fronte all'esigenza di confrontarsi con la "storia" della propria letteratura, il poeta esorta le nuove generazioni a riflettere sull'importanza della tradizione (e quindi, paradossalmente, dello stesso Petrarca), precisando, però, che una tale operazione "non è un invito di ritorno all'intelligenza di ogni manifestazione trascorsa dello spirito della propria terra. È qualcosa di più profondo di una discesa inerme in alcune forme di unanime consenso" (16). Una precisazione cautelativa, si potrebbe dire, nei confronti di quei "parnassiani" che concepiscono la tradizione come un repertorio di schemi, e che conferma come Quasimodo, pur non avendo ancora individuato un'alternativa, ribadisca la sua insoddisfazione per il sistema poetico italiano. $\mathrm{Ma}$, al di là della solita frecciata rivolta all'ermetismo e ai suoi cultori critici, siamo anche di fronte al primo passo inconscio verso la soluzione del problema (come lo intende Quasimodo, ovviamente). Qui l'accenno è diretto a Petrarca, il poeta che ha involontariamente gettato le basi per quelle biasimate "forme di unanime consenso". Ma un approccio simile alla tradizione, basato più sulla comprensione che l'imitazione, potrebbe valere per chiunque, se solo si definissero le ragioni estetiche che ci spingono, appunto, a richiamarci ad essa. E Quasimodo, pur non potendosi 
ancora riferire a Dante, lascia comunque intravedere dove lo condurrà la sua ricerca:

Io non credo alla poesia come "consolazione" ma come moto ad operare . . in seno alla vita, cioè dentro l'uomo . . . . Il poeta parla della società in cui vive, grida se deve gridare. (18)

Quando scrive queste parole, Quasimodo ha già cominciato a "gridare" con quelle poesie che, composte durante e subito dopo la fine della guerra, escono proprio nel "46 a Milano per i "Quaderni di Costume" con il titolo di Con il piede straniero sopra il cuore (si tratta di gran parte dei componimenti che l'anno successivo confluiranno in Giorno dopo giorno). E che Dante abbia contribuito al tono di queste poesie, lo prova la lirica più nota di questa raccolta, Alle fronde dei salici, il cui ultimo verso "oscillavano lievi al triste vento", non pare esente da un contatto con il famoso verso del quinto canto dell'Inferno: "e paion sí al vento esser leggieri". Siamo in ambito infernale, ma non è il Dante petroso cui Quasimodo si appellerà più avanti, si potrebbe obiettare. Ed è vero, ma in altre prove dello stesso libro le riprese dantesche sono in tono con quell'inferno "reale" prediletto da Quasimodo. Spogli e riscontri vari sono stati già effettuati, come s'è detto (cfr. Scorrano 40 sgg.; Ciccarelli 417). Qui si vuole sottolineare, però, come non conti solo la pura ripresa lessicale (anche nella stessa Alle fronde dei salici la parola "ghiaccio", dato il contesto - "fra i morti abbandonati nelle piazze / sull'erba dura di ghiaccio" - lascia pensare al ripetuto uso che Dante ne fa nell'Inferno) bensí anche la connotazione negativa che accompagna tali riprese. Un esempio su tutti: il termine "cerchio" che nella poesia Dalla rocca di Bergamo alta ricorda, complici anche altri precisi riferimenti infernali, la struttura stessa dell'inferno dantesco: "Eri nel cerchio ormai di breve raggio: / e tacquero l'antilope e l'airone / persi in un soffio di fumo maligno". La ripresa non potrebbe essere più peculiare: l'avanzare del male causato dall'inferno bellico è direttamente proporzionale, se possibile, al procedere concentrico del mondo senza speranza di Dante. E in un'altra lirica, Neve, il "cerchio bianco dei sepolti" rinvia di nuovo, per il senso (i morti seppelliti dalla coltre di neve) e per l'immagine, a quel cerchio di breve raggio, l'infernale lago di Cocito.

La parola realistica di Dante, malgrado le difficoltà teoriche, già opera nelle poesie pubblicate nel ' 46 , quindi. Ma la chiave di volta che spalanca a Quasimodo la possibilità d'individuare pubblicamente in Dante quel maestro di cui le nuove generazioni hanno bisogno, è racchiusa nella definizione di ciò che che per lui dovrebbe essere il tratto distintivo di ogni poeta: “Ogni poeta - scrive infatti Quasimodo sempre nel '46- ... si riconosce non soltanto dalla sua voce ritmica o interna ma soprattutto dal suo linguaggio" (15-6). Abbiamo anticipato come Quasimodo abbia sostenuto che sono le "ragioni formali", cioè il linguaggio, a presentarci una determinata impostazione estetica e, quindi, i "contenuti". L'aggancio teorico con 
Dante, sebbene ancora sotterraneo, è ormai avvenuto. ${ }^{12}$ Perché venga alla luce dichiaratamente bisognerà aspettare qualche anno, come si sa. Forse Quasimodo per acquistarne coscienza dovrà prima passare attraverso l'esperienza delle proprie poesie infernali. Forse avrà anche bisogno di verificare, attraverso una serie di parallelismi che poi-nel vivo delle polemiche degli anni '50-diverranno pure strumentali, come il dialogo appreso da Virgilio andasse in qualche modo rivestito della concretezza infernale dantesca per avvicinarsi allo scopo "visivo" e "diretto" desiderato. Ed anche se Quasimodo arriva a Dante per via indiretta, per esclusione, dopo aver sperimentato il fallimento altrui (Petrarca), crediamo non si possa negare la sincerità del movente che spinge il siculo-greco ad abbandonare il suo mondo di simboli lirici, per misurare la propria esperienza con il poeta che ci ha insegnato ad individuare nella poesia il reale dell'uomo.

\section{Indiana University}

\section{NOTE}

1 Nell'impossibilità di stilare liste che comunque risulterebbcro parziali e incomplete, qui si possono ricordare i nomi di quattro autori che, fra i poeti della generazione subito precedente e immediatamente posteriore a Quasimodo, si sono soffermati sul problema con maggiore altenzione: Ungaretti, Montale, Luzi c Bigongiari.

2 A parte che in duc saggi sull'Alighieri (Dante, '52; Brunetto Latini, '57), Quasimodo rifiette sul ruolo svolto da Dante all'interno del panorama letterario italiano in diversi interventi dedicati a questioni di poetica novecentesca: Pocsia contemporanea ('46), Discorso sulla poesia ('53) e Il poeta e il politico ('59).

3 E diciamo "tarda" perché Quasimodo, se si escludono le prefazioni allc traduzioni dei classici (lirici greci, Virgilio ed Eschilo) ed un breve ma significativo scritto del '39 (D'Annunzio e noi) comincia ad interessarsi di teoria e di critica letteraria soltanto dopo il ' 45.

4 È questa la famosa definizione coniata da Oreste Macrì nel suo studio del 1938 su Quasimojo. Il poeta siciliano, almeno a partire dal ' 45 , ha sempre mostrato una certa insofferenza per questa tcoria che è, forse, indirettamente e parzialmente responsabile per le polemiche anti-ermetiche in cui Quasimodo si è lasciato coinvolgere nel tentativo di svincolarsi da quello status di "lirico puro" implicito nella definizione. Una situazione che ha finito per ingigantire ed esasperare le pur indubbic trasformazioni realistiche avvenute nelle poesie del dopogucrra. Si riflctta, in proposito, su questa asserzione apologetica dell'autorc: "Non mi si fraintenda: 'parola' ha per me, ha sempre avulo, significalo anche di epica, di drammatica" (15).

5 Più che quelle di Giorno dopo giorno del '47, cfr. La vita non è sogno (1949) e la sezionc "Quando caddero gli alberi e le mura" di ll falso e vero verde del '57.

6 È risapulo che questo passaggio dall'ermetismo al realismo, ha dato vita alla querelle sui due Quasimodo. Parte della critica ha sempre valutato questo mutamento come una maturazione della poetica quasimodiana; altri, invece, tendono a valutarc negativamente qucllo che è visto come un facile passaggio alle poetiche in voga nel dopogucra (su questo problema si vedano le equilibrate osservazioni di Bo, Prefazione).

7 È fuor di dubbio che Quasimodo, sulla scia del suo approccio svolto in chiave contemporanca dell'opera dantesca, abbia soltoscritto "un giudizio recepito in modo inerte e ripetuto senza procedere a verifiche sul campo" (Scorrano 35). Quasi a controcanto (voluto?) di questa rigida c poco accurata posizione idcologica, si pensi invece a quanlo ha sostenuto 
Montale: “. . . chi ha il senso della poesia non tarda ad accorgersi che Dante non perde mai la sua concretezza neppure nei casi in cui la fabbrica si mostri meno rivestita della supposta efflorescenza" (28).

8 È stato più volte sottolineato dalla critica come gli interventi saggistici di Quasimodo servano più a giudicare il suo pensiero e $\mathrm{i}$ suoi intenti poetici, che non come reale contributo critico sugli autori o le correnti di volta in volta analizzati. E questo, dati i presupposti del suo recupero dantesco, vale in misura ancora maggiore per gli scritti sull'Alighieri.

9 Era inevitabile che Quasimodo, data la polemicità del suo sforzo anti-tradizionale (e quindi anti-elegiaco), finisse per puntare l'obiettivo delle sue critiche sull'indiretto "responsabile" dello scarso realismo della poesia italiana. È necessario precisare, comunque, che se è vero che nel Discorso sulla poesia il poeta arriverà a suggerire di "leggere Dante per dimenticare Petrarca" (45), altrove specificherà anche, però, come le sue critiche s'appuntino contro il "mormorio petrarchesco" degli imitatori, incapaci di rifarsi al Petrarca "esemplare di Leopardi” (124). E forse, ma la si prenda come pura ipotesi, chi asseriva tali frasi nel '52, suggeriva indirettamente che fra i pochi capaci di ripetere l'esperienza petrarchesca senza scadere nella banalità dell'imitazione esterna si doveva contare l'autore di Acque e Terre.

10 Proprio l'occasione del Nobel aveva risollevato le polemiche intorno alla sincerità o meno (finendo per offuscare lo studio sul reale valore estetico delle poesie) della cosiddetta stagione civile di Quasimodo. Per avere un'idea dell'asprezza di tali polemiche penso sia sufficiente ricordare come uno dei maggiori critici italiani del secolo, Emilio Cecchi, avesse sarcasticamente salutato il conferimento del premio a Quasimodo, intitolando un suo articolo sul Corriere della sera, "A caval donato non si guarda in bocca ... .".

11 Per queste lettere cfr. Lajolo.

12 E di un Dante "sotterraneo", che si propone silenziosamente alla cultura novecentesca, aveva parlato Quasimodo nel saggio sull'Alighieri del '52 (121).

\section{OPERE CITATE}

Bárberi Squarotti, Giorgio. "La critica Quasimodiana”. Quasimodo l'uomo e il poeta. Ed. Rosa Brambilla. Assisi: Cittadella Editrice, 1983: 166-84.

Bo, Carlo. "Dante e la poesia italiana contemporanea". Terzo Programma 4 (1965): 193-8.

Prefazione. Salvatore Quasimodo. Poesie e Discorsi sulla poesia. Ed. Gilberto Finzi. Milano: Mondadori, 1971: ix-xviii.

Ciccarelli, Andrea. "Quasimodo e Dante". Salvatore Quasimodo. La poesia nel mito e oltre. Ed. Gilberto Finzi. Bari: Laterza, 1986. 411-24.

Jacomuzzi, Angelo. "Alcune premesse per uno studio sul tema Montale e Dante". In Zennaro. 217-27.

Lajolo, Davide, ed. Quasimodo. Lettere d'amore a Maria Cumani. Milano: Mondadori, 1973.

Luzi, Mario. L'inferno e il limbo. Milano: Il Saggiatore, 1964.

Macrì, Oreste. "La poetica della parola di Salvatore Quasimodo". Prefazione. Salvatore Quasimodo. Poesie. Milano: Primi Piani, 1938.

Montale, Eugenio. "Dante ieri e oggi”. Sulla Poesia. Ed. Giorgio Zampa. Milano: Mondadori, 1976.

Petrucciani, Mario. "Due paragrafi per Dante e il Novecento. I. Tra le due guerre: dalla 'Ronda' all'ermetismo". In Zennaro. 163-86.

Quasimodo, Salvatore. Il poeta e il politico e altri saggi. Milano: Mondadori, 1967.

Scorrano, Luigi. "Dante 'sotterraneo' e dantismi nella poesia di Quasimodo". Otto/Novecento 4-6 (1988): 33-60.

Ungaretti, Giuseppe. Vita d'un uomo. Saggi e interventi. Eds. Mario Diacono e Luciano Rebay. Milano: Mondadori, 1974.

Zennaro, Silvio, ed. Dante nella letteratura italiana del Novecento. Roma: Bonacci, 1979. 